\title{
Knowledge of Morphine Administration by Ambulance Paramedics: A Comparison of Two Clinical Levels of Paramedics
}

\author{
Anthony Weber ${ }^{1}$, Brian Maguire1, Trudy Dwyer ${ }^{2}$ \\ ${ }^{1}$ School of Medical and Applied Sciences, Faculty of Higher Education, CQ University, Rockhampton, Australia \\ ${ }^{2}$ School of Nursing and Midwifery, CQ University, Rockhampton, Australia \\ Email: *a.weber2@cqu.edu.au
}

Received 15 October 2015; accepted 12 December 2015; published 15 December 2015

Copyright (C) 2015 by authors and Scientific Research Publishing Inc.

This work is licensed under the Creative Commons Attribution International License (CC BY). http://creativecommons.org/licenses/by/4.0/

(c) (i) Open Access

\section{Abstract}

Objective: The purpose of this study is to identify any differences in adherence to morphine administration protocols between two clinical levels of paramedics within one emergency medical system in Australia. Methods: The participants were made up of 63 Advanced Care Paramedics (ACPs) who were trained in basic and intermediate life support and 45 Intensive Care Paramedics (ICPs) who were trained in advanced life support skills; all were operational and practicing paramedics qualified in morphine administration. Participants were required to complete a survey designed to assess their knowledge of morphine drug therapy protocol (DTP). The survey involved two sections: 1) demographics, and 2) questions related to two scenario-based cases. Scenario one was a cardiac case and scenario two was a pediatric case. Results: In scenario one, more intensive care paramedics administered the correct dose than the advanced care paramedics $(25.4 \% \mathrm{vs} .4 .4 \%$; $d f=101, P=0.002)$; scenario two showed no statistically significant difference between the two groups $(35.5 \%$ vs. $31.6 \%$; $d f=97, P=0.3)$. Conclusion: The comparison of the two levels of paramedics and their adherence to drug protocols identified that ICPs had a higher rate than ACPs for adherence to morphine administration protocols for patients suffering cardiac related chest pain. The clinical differences may be based on the length of the opioid-administration training program, clinical exposure to morphine administration, work experience and length of qualifications as an ACP or ICP. Our findings suggest that there is a need for structured initial and ongoing continuing education programs in pain management to maintain knowledge and behavior in pain management strategies. Further research is also required into paramedic's behavioral intention regarding morphine administration to also identify what constructs affect their intent to administer morphine.

${ }^{*}$ Corresponding author. 
Keywords

Pain Management, Advanced Care Paramedics, Intensive Care Paramedics, Clinical Judgement

\section{Introduction}

Pain is a common symptom that leads people to seek health care both in the prehospital and hospital environments [1]-[3]. While many health disciplines are involved in pain management, paramedics play a pivotal role in the assessment, evaluation, early intervention and management of many patients suffering pain. Pain management is dependent on the attending paramedics' pharmacological knowledge, protocol familiarity and on the timely initiation of opioid drugs.

Weber et al. described the paucity of high-quality published evidence with which to "validate many aspects of pain management in paramedic practice" (2013: p. 454) [4]. Inadequate or under medications have been identified as undesired outcomes when the health professional's knowledge and assessment skills are below standard [5] [6]. There are a number of possible causes for inadequate knowledge and assessment skills including organisational culture and lack of ongoing education of health professionals [5] [7]. In response, some Australian ambulance services have implemented problem-based education and practical assessments that follow an audit process [8]. However, programs such as these have had limited success increasing knowledge application or changing clinician behavior in regards to pain relief when no on-going education processes have been implemented [5] [7].

An insufficient amount of time allocated to health professionals pain management education and training has been identified as a barrier to the administration of medications such as opioids [9] [10]. Educators in the medical field have found that increasing clinicians' knowledge of pain management through effective, high quality education and continuing education programs [11] eliminates this barrier [12] [13]. A study conducted by French et al. (2013) implemented and evaluated a continuing education pain management program for paramedics [14]. The authors concluded that a program that was three hours long was sufficient to significantly improve the paramedic's pain management knowledge and behavior [14].

Pain management strategies also require effective education and continuing education including the use of narcotics in pain management and the use of non-narcotic interventions [11]. In pain management cases paramedics should be equipped with appropriate attitudes [7] and effective clinical judgment to utilize methods of pain relief other than pharmacological interventions [11]. A number of other studies have shown the benefits of education and/or guidelines on improved prescribing patterns both in general terms and specifically for non steroidal anti-inflammatory drugs (NSAID), paracetamol and pethidine [1]. For a successful pain management program to be installed into ambulance services, an effective and successful program must firstly be introduced through paramedic baccalaureate degree programs and then by the ambulance services through in-service education. Just as important is the follow-up of clinical practices by paramedics through a quality assurance program that includes call review and continuing education [4] [15] [16].

While many paramedic programs continue to deliver pain management programs that vary in duration [7], the optimal length of the pain management program to ensure the transference of knowledge into clinical practice is not known. No comprehensive research has been found that describes the different levels of education of paramedics and the administration of pain analgesia.

The Australian Emergency Medical Service (EMS) system in this current paper has implemented different pain management programs to meet the needs of varying education level of paramedics, including intensive care paramedics (ICP) who provide advanced life support versus advanced care paramedics (ACP) who provide primarily basic life support and some limited advanced life support procedures (all ICPs are former ACPs who then graduate from an advanced clinical program). These two levels of paramedics are consistent with the two tier level for scope of practice in ambulance services across Australia [17]. The ICPs received 40 hours of on-going pharmacology education over one semester in postgraduate studies; the ACPs received a single eight hour pain management training package that was administered by regional coordinators.

The aim of this study is to identify if there is a relationship between duration of pain management training programs and adherence to pain management protocols between the two levels of paramedics. The findings may 
be used to improve organisational awareness of factors that contribute to the future education and professional development of paramedics.

\section{Methodology}

A cross-sectional non-experimental survey design was employed for this study. Data were collected by a selfreport on a web-survey (electronic questionnaire). Scenario based questions were used to measure the paramedics' knowledge of morphine drug doses for patients with pain. The scenarios provided to the paramedics were validated when used in the education of advanced care paramedics in a pain management workshop [8].

\subsection{Subjects}

The target population for this study was practicing intensive care (ICP) and advanced care paramedics (ACP) who still retain their scope of practice for administration of morphine in the prehospital setting in one Australian state. As the delivery of the pain management package for ACPs was different between different regions in the state, the advanced care paramedic sample group came from one region only (all the ICPs in the state received identical training). The sample consisted of: the advanced care paramedic (ACP) ( $n=63)$ within one region of the state; and, all intensive care paramedics (ICP) $(n=45)$ throughout the state. The survey-return rate yielded a response of $35 \%$ of the overall population.

\subsection{Measures}

The self-administered on-line survey consisted of a demographic and a scenario-based knowledge section comprising two case scenarios on pain management (Table 1). Participating paramedics selected the morphine administration dosage that best reflected their behavior in the clinical setting. Individual responses were scored and compared against the ambulance service's standard drug therapy protocols [8]. Comparisons of scores between the two groups were made using independent t-test for parametric data and chi-square test for non-parametric data. A P value of less than 0.05 was set for the study. De-identified demographic data and scores were entered into and evaluated using SPSS 17.0 (SPSS, 2009) statistical package software.

\subsection{Ethical Considerations}

This study received approval from the CQ University human research ethics committee (approval number H08/ 10-058) and the Australian Ambulance Service.

\section{Results}

Of the 108 paramedics who chose to participate in the study (Table 2), the majority were male $(87,81 \%)$ and advanced care paramedics $(63,58 \%)$. The range of respondents' experience in paramedic practice within the ambulance service was from one to over 20 years (mean 12.0 years; SD $=6.2$ ) with $18 \%(n=17)$ of the respondents having over 20 years experience. The ICP group was more experienced with an average of 8.5 years (SD 4.2) in their current role, compared to 5.1 years (SD 2.7) for the ACP group. A difference was also evident in relation to career length where ICPs on an average had been in the profession for 15 years (SD 5.34) and the ACPs just under 10 years (SD 5.8). Educational qualifications of the ACP varied with 69 percent $(n=37)$ possessing either an Associate Diploma or Diploma, a fifth $(\mathrm{n}=11)$ with a Bachelor's degree and the remainder

\section{Table 1. Scenario based assessment of knowledge.}

\section{Scenario 1}

You are called to a motel room where you find a 45-year-old male patient complaining of central chest pain radiating down his left arm, described as severe 10/10 pain. The patient states to you that he is not sure if it is relevant but he has taken Viagra one hour ago. The patient's vital signs are within normal parameters for narcotic analgesia.

\section{Scenario 2}

You have been dispatched to a four-year-old female who has fallen down three stairs. She is conscious and alert with pain in the left arm. The patient's father states she slipped down 3 stairs and did not hit her head. The patient appears to have a fractured left humerus. The patient is showing signs of significant pain and vital signs are within normal parameters for narcotic analgesia 
Table 2. Demographics of participants.

\begin{tabular}{|c|c|c|c|c|c|c|c|c|c|}
\hline \multirow[t]{2}{*}{ Sample } & \multicolumn{2}{|c|}{$\begin{array}{l}\text { Advanced Care Paramedic } \\
(\mathrm{n}=63)\end{array}$} & \multirow[b]{2}{*}{ SD } & \multicolumn{3}{|c|}{$\begin{array}{l}\text { Intensive Care Paramedic } \\
(\mathrm{n}=45)\end{array}$} & \multicolumn{3}{|l|}{$\begin{array}{l}\text { Total } \\
(\mathrm{n}=108)\end{array}$} \\
\hline & n (\%) & M & & n (\%) & M & SD & n (\%) & M & SD \\
\hline \multicolumn{10}{|l|}{ Career length } \\
\hline & $52(83)$ & 9.7 & 5.84 & $39(87)$ & 15.1 & 5.34 & $91(84)$ & 12.4 & 6.22 \\
\hline \multicolumn{10}{|l|}{$\mathrm{P}=0.001$} \\
\hline \multicolumn{10}{|l|}{ Gender } \\
\hline Female & $16(25.8)$ & & & $4(8.9)$ & & & 20 (18.7) & & \\
\hline Male & $46(74.2)$ & & & 41 (91.1) & & & 87 (81.3) & & \\
\hline \multicolumn{10}{|l|}{$P=0.027$} \\
\hline \multicolumn{10}{|l|}{ Qualified Length } \\
\hline & $52(83)$ & 5.1 & 2.73 & $39(87)$ & 8.5 & 4.27 & $91(84)$ & 6.5 & 3.85 \\
\hline \multicolumn{10}{|l|}{$P=0.001$} \\
\hline \multicolumn{10}{|l|}{ Career position } \\
\hline Operational & 57 (98.3) & & & $24(66.7)$ & & & $81(86.2)$ & & \\
\hline Educator/CSO & $1(1.7)$ & & & $7(19.4)$ & & & $7(7.4)$ & & \\
\hline Manager & & & & $5(13.9)$ & & & $6(6.4)$ & & \\
\hline \multicolumn{10}{|l|}{$P=0.001$} \\
\hline \multicolumn{10}{|l|}{ Highest Qualification } \\
\hline Associate Diploma & $18(34)$ & & & & & & $18(21.4)$ & & \\
\hline Diploma & 19 (35.8) & & & & & & $19(22.6)$ & & \\
\hline Advanced Diploma & $2(3.8)$ & & & $5(16.1)$ & & & $7(8.3)$ & & \\
\hline Bachelor & $11(20.8)$ & & & $14(45.2)$ & & & 25 (29.8) & & \\
\hline Graduate Certificate & $2(3.8)$ & & & $2(6.5)$ & & & $4(4.8)$ & & \\
\hline Graduate Diploma & $1(1.9)$ & & & $8(25.8)$ & & & $9(10.7)$ & & \\
\hline Masters & & & & $1(3.2)$ & & & $1(1.2)$ & & \\
\hline Other & & & & $1(3.2)$ & & & $1(1.2)$ & & \\
\hline$P=0.001$ & & & & & & & & & \\
\hline
\end{tabular}

(11\%) having graduated from post-graduate programs. In comparison almost half (45\%) of the ICP's had completed a Bachelor degree or a graduate diploma ( $>25 \%)$. One ICP had completed a master's degree.

The respondents identified how many times (on average) they administer morphine during their routine duties. Nine of the one hundred and eight participants had never administered morphine in their daily duties, with ACPs making up the majority of these (6.5\% vs. $1.9 \%)$. Most $(n=95,88 \%)$ participants had administered morphine between 1 and 3 times a shift with the ICP making up the majority of this cohort (51.4\% vs. 37.4\%). Very few (n = $3,<2 \%$ ) of the participants administer morphine four or more times a shift with the ICP being the only clinical level administering these doses $(\mathrm{P}=0.134)$.

\subsection{Survey Results}

The survey for scenario 1 was developed from a standard drug therapy protocol (DTP) of morphine administration for ACPs and ICPs. The second scenario focused on the management of a paediatric patient suffering significant pain. The second scenario, like the first, identified the respondent's knowledge of current DTP drug dos- 
ages. To determine the respondent's knowledge of drug therapy protocols as per the ambulance service's DTP, information pertaining to the correct drug dose was scored by either the correct dose, incorrect dose or no dose (as shown in Table 3).

\subsubsection{Scenario One}

The majority of ACP respondents $(45 / 63,71 \%)$ to scenario one would administer morphine using the correct DTP. There were 25\% (16/63) that administered the incorrect DTP of $2.5 \mathrm{mg}$ with three percent of respondents (2/63) stating they would not administer morphine. In relation to the ICP respondents; 77\% (35/45) stated they would administer the appropriate doses as per their DTP. Approximately $4 \%(2 / 45)$ administered outside of their DTP and a further $13 \%(6 / 45)$ would not administer morphine $(d f=101, \mathrm{P}=0.002)$.

\subsubsection{Scenario Two}

For this scenario over half of the ACP respondents (58\%, 33/63) recognised the correct commencing dose of morphine for paediatric patients. Thirty two percent (18/63) of the ACP respondents did not indicate the correct dose, either through administering too high a dose or opting for smaller aliquots of morphine. Six of the ACP respondents $(10 \%)$ opted to not administer the morphine.

Of the ICP respondent group, 46\% (21/45) would have administered the correct dose with a further $35 \%$ (16/45) administering a dose outside of the protocols; $13 \%$ (6/45) of ICP respondents made the judgement to not administer morphine $(\mathrm{P}=0.30)$.

\section{Discussion}

Adherence to protocols is required for the safe and effective management of patients and in particular when administering a narcotic analgesia. This study identified that intensive care paramedics adhered to drug therapy protocols for morphine administration for patients suffering cardiac related chest pain which may have a positive effect on patient care outcomes. When it came to drug dose regimes for paediatric pain management, the intensive care paramedics showed more drug errors than the advanced care paramedics but the results were not statistically significant.

The findings of this study identified that there are statistically significant differences between the adherences to protocol for morphine administration by two different clinical levels of paramedics.

Variables that may affect these outcomes include the span of experience of the paramedics; the length of qualification, the subject's clinical training, the exposure to morphine administration and length of the morphine education programs.

A possible influence on outcomes may be that the ICP group had received on-going education on the topic of pain management and morphine administration. Given the correlation between ongoing education [11] and behaviour [7] this may well have influenced the pain management practices of the ICPs.

Table 3. Morphine administration knowledge by 2 paramedic groups.

\begin{tabular}{cccc}
\hline & ACP $(\mathrm{n}=63)$ & ICP $(\mathrm{n}=45)$ & Total $(\mathrm{n}=108)$ \\
\cline { 2 - 3 } & $\mathbf{n}(\%)$ & $\mathbf{n}(\%)$ & P-Value \\
\hline Scenario One & & & 0.002 \\
Correct DTP & $45(71.4)$ & $35(77.8)$ & 0.002 \\
Incorrect DTP & $16(25.4)$ & $2(4.4)$ & 0.001 \\
No administration & $2(3.2)$ & $6(13.3)$ & 0.30 \\
2 ICP did not respond & & & 0.30 \\
Scenario Two & $33(57.9)$ & $21(46.6)$ & 0.001 \\
Correct DTP & $18(31.6)$ & $16(35.5)$ & $6(13.3)$ \\
Incorrect DTP & $6(10.5)$ & & \\
No administration & & & \\
2 ICP did not respond & &
\end{tabular}


Emerging pain control is a very challenging area for paramedics. Over the past decade Hennes (2006) identified that several published studies have documented significant deficiencies in prehospital pain management [3] [18]. Given the front line positioning of emergency services, paramedics are well placed to become active leaders and primarily responsible for reducing human pain and suffering. Reducing the gap between contemporary pain management research and paramedic care has been a focus for many organisations including the Australian and New Zealand College of Anaesthetists (ANZCA) [1].

A descriptive and comparative statistical analysis was performed in an Australian emergency department (ED) pain management study. The majority of their patients arrived by ambulance $(\mathrm{n}=1966,95 \%)$. The analgesia provided to patients by paramedics was morphine $(n=120,14.2 \%)$, morphine derivatives $(n=55,6.5 \%)$, or methoxyflurane $(n=247,29.3 \%)$. Their findings observed that the majority of cohorts presented to the ED were still in significant pain [19]. These findings concur with international literature which identified that patients presented to ED's in the United States, France and the United Kingdom continued to report significant pain and only $49.3 \%$ of these patients had received opioid analgesia [3]. A survey of ambulance services in the United Kingdom identified that only eight percent of paramedics gave analgesia other than entonox; two of the influencing factors were the skill and education of the paramedics [20]. These findings are consistent with our findings where we identified that the intensive care paramedics received 40 hours of education in pain management compared to a single eight-hour continuing education package for the advanced care paramedic.

It has been documented that a lack of education for paramedics in pain physiology, pain assessment and opioid pharmacokinetics are contributing factors to oligoanalgesia [3]. Two studies discussed the impact of education on pain management in the prehospital setting. In a study from France, a quality control program focussing on pain treatment and the implementation of a pain management protocol, significantly improved pain management to patients [21]. Lovesky, Bird and Restoccia (2003) identified that a prehospital pain management training program increased the number of patients receiving pain medication three fold [12]. Further, paramedics increased their knowledge of pain principles by $17.5 \%$ following participation in an education program and were $32.2 \%$ more likely to provide non-pharmacologic therapy and were $24 \%$ more likely to document findings when introduced to a continuing education program [11]. They not only identified that paramedics significantly improved their basic knowledge but more importantly the underpinning knowledge behind clinical practice was shown to improve with enhanced knowledge of neuroanatomy [11] [22].

Interestingly, studies that have examined the effects of nursing education programs have found that one continuing education class is often not effective in increasing knowledge [10]. In a study of nurses' knowledge (post nursing course) of children's pain management only $50 \%$ to $77 \%$ of nurses responded correctly to questions on pain management. Nurses who continued to attend paediatric pain management sessions went on to administer more opioids than those who did not [6].

A survey into pain management found that $76 \%$ of physicians surveyed $(n=682 / 897)$ believed that their limited patient-pain assessment skill was the single most important barrier to adequate pain management [23]. As a result, their reluctance to prescribe opioids may be due, in part, to their limited training, which was cited as the second most important barrier among $61 \%$ of the respondents. Researchers concluded that proper knowledge of the assessment of pain, the management of side effects, and better understanding of opioid analgesics were needed [24].

A study into aspirin administration rates in Oregon Emergency Medical Technician (EMT) services showed that two simple consecutive educational sessions increased the rate of aspirin administration from $15.1 \%$ to $37 \%$ for patients with Acute Coronary Syndrome (ACS) [25]. The researchers found that while there was resistance to the 30 minute lecture, knowledge increased and improved aspirin administration occurred [25]. The two brief educational interventions; a protocol change and a lecture, led to modest increases in aspirin administration rates.

Rickard et al. (2007) used a randomised, controlled, open-label study to compare the use of intranasal fentanyl (INF) $(\mathrm{n}=127)$ to intravenous morphine (IVM) $(\mathrm{n}=100)$ for prehospital analgesia. All paramedics received education at the commencement of the study. While INF was found to be an effective alternative to IVM for use in the prehospital setting it was the paramedics' attitudes towards the change that was of interest to the researcher more so than the education undertaken [26]. The researchers identified that this program enhanced the knowledge of the paramedics and improved their attitude towards pain management. This study is consistent with other studies which have identified that in-service education programs for paramedics not only improve patient care outcomes [25] [27] but also greatly enhance the impact of knowledge and skills on the overall quality 
of care [11] [15] [28].

Introducing new clinical practice guidelines (CPG), clinical practice procedures (CPP) and drug therapy protocols (DTP) into ambulance organisations may have little effect on clinical practice unless the dissemination is supported by quality pain management education and continuing education programs.

This study reviewed the difference in behavior between the two levels of paramedics within an Australian Ambulance Service. The study highlights the need for ongoing continuing education programs to be implemented as well as a review into the behavioural aspects and constructs of paramedics in administering morphine. A study by Hennes and Kim (2006) supports this finding by identifying that a knowledge deficit of paediatric pain management among paramedics in the United States may be a significant barrier for improving pain management; while $93 \%$ had knowledge that analgesia is indicated for adults, only $50 \%$ stated that analgesia is indicated for children with extremity fractures [3].

The outcomes of this paper were to identify if there is a relationship between duration of pain management programs and adherence to protocols set out by an Australian Ambulance Service. We found that there was a statistically significant difference in correct morphine administration rates between ACPs and ICPs. The findings suggest that education and continuing education of all paramedics is required for adherence to drug therapy protocols and is consistent with findings from other studies. The length of education contributes to the paramedic's ability to accurately administer the correct doses of a drug to patients suffering significant pain.

\section{Limitation}

The present study has the following limitations: As with many surveys, because of the low response rate (35\%) and limited sample based on only one cohort of ACPs within one region of a large ambulance service, data from the questionnaire should be interpreted with caution. Moreover, since this was a voluntary survey, results may be skewed and may not be representative of all advanced care and intensive care paramedics.

Another limitation is the difference of experience between the two groups of participants; this includes the length of clinical experience, length of employment and, academic education. The intensive care paramedics were more experienced in paramedic practice, more clinically experienced and held higher academic education qualifications, which may have affected the results.

Due to the sensitive nature of the topic, as outlined by one ICP, bias may have occurred which may have led respondents to modify their actual responses in order to demonstrate that they are using clinical judgement rather than following protocols.

Replicating this study using a larger population and different clinical practice regimen is recommended. One study is not conclusive as to the general measure of paramedics' attitude towards morphine administration and a broader base is needed for comparison.

\section{Recommendations}

Based on the findings from this study, the following recommendations are proposed to enhance pain management within ambulance services:

1) Distribute current National Health and Medical Research (NHMRC) pain management guidelines to all paramedics.

2) Include current pain management information in all baccalaureate programs, paramedic in-service programs and orientation programs.

3) Implement an annual in-service continuing education pain management program.

4) Conduct on-going patient-centred studies to assess paramedic knowledge and behavioural intention versus actual behaviour regarding pain and pain management.

\section{Conclusions}

The aim of the study was to identify if there was a relationship between duration of pain management programs and adherence to pain management protocols between two levels of paramedics from one Australian Ambulance Service. The two levels of paramedics were Advanced Care Paramedics who were trained in basic and intermediate life support and Intensive Care Paramedics who were trained in advanced life support skills. Although morphine as a drug therapy protocol is part of the annual skills validation, pain management validation processes do 
not exist within the service. This study identified that adherence to drug doses improved with more time applied to the education of a paramedic and more exposure to education. Pain management should therefore be considered a part of annual competency evaluations.

Educational interventions that have successfully increased a paramedic's knowledge may fail to have a significant impact on a paramedic's behaviour. Often pain management education must be continually reinforced in order to change behaviour. A paramedic's knowledge, regarding pain management with specific regard to opioid administration, can significantly influence the treatment and management of patients with pain.

Further research into paramedic's knowledge and behavioural intention, regarding morphine administration, is required. Implementation of pain management content into paramedic program curricular not only improves declarative and procedural skills to undergraduate students, but also improves the scope of practices for all levels of paramedics when introduced as continuing medical education programs. This continuum of education including introducing best practices will enhance further learning and better prepare paramedics for enhanced clinical practice. Finally, continued morphine administration research is required to explore why discrepancies occur among education, protocol and practice.

\section{References}

[1] Australian and New Zealand College of Anaesthetists and Faculty of Medicine (2005) Acute Pain Management: Scientific Evidence. 2nd Edition, Australian Government National Health and Medical Research Council, Canberra.

[2] Galinski, M., Ruscev, M., Gonzalez, G., Kavas, J., Ameur, L., Biens, D., Lapostolle, F. and Adnet, F. (2010) Prevalence and Management of Acute Pain in Prehospital Emergency Medicine. Prehospital Emergency Care, 14, 334-339. http://dx.doi.org/10.3109/10903121003760218

[3] Hennes, H. and Kim, M. (2006) Prehospital Pain Management: Current Status and Future Direction. Clinical Paediatric Emergency Medicine, 7, 25-30. http://dx.doi.org/10.1016/j.cpem.2006.01.008

[4] Weber, A., Dwyer, T. and Maguire, B. (2013) Appropriate Morphine Administration by Paramedics: A Review of the Literature. Journal of Paramedic Practice, 5, 454-460. http://dx.doi.org/10.12968/jpar.2013.5.8.454

[5] Chiang, L., Chen, H. and Huang, L. (2006) Student Nurses’ Knowledge, Attitudes, and Self-Efficacy of Children’s Pain Management: Evaluation of an Education Program in Taiwan. Journal of Pain and Symptom Management, 32, 82-89. http://dx.doi.org/10.1016/j.jpainsymman.2006.01.011

[6] Clarke, E., French, B., Bilodeau, M., Capasso, V., Edwards, A. and Empoliti, J. (2006) Pain Management Knowledge, Attitudes and Clinical Practice: The Impact of Nurses' Characteristics and Education. Journal of Pain and Symptom Management, 11, 18-31.

[7] Weber, A., Dwyer, T. and Mummery, W.K. (2012) Morphine Administration by Paramedics: An Application of the Theory of Planned Behavior. Injury: International Journal of the Care of the Injured, 43, 1393-1396.

http://dx.doi.org/10.1016/j.injury.2010.12.006

[8] Queensland Ambulance Service (2007) Pain Management Package.

[9] Lord, B.A. and Parsell, B. (2003) Measurement of Pain in the Prehospital Setting Using a Visual Analogue Scale. Prehospital and Disaster Medicine, 18, 353-358. http://dx.doi.org/10.1017/S1049023X0000131X

[10] Dalton, J., Blau, W., Carlson, J., Mann, J., Bernard, S., Toomey, T., Pierce, S. and Germino, B. (1996) Changing the Relationship Among Nurses’ Knowledge, Self-Reported Behaviour, and Documented Behaviour in Pain Management: Does Education Make a Difference. Journal of Pain and Symptom Management, 12, 308-319. http://dx.doi.org/10.1016/S0885-3924(96)00183-2

[11] French, S.C., Chan, S.B. and Ramaker, J. (2013) Education On Prehospital Pain Management: A Follow-Up Study. Western Journal of Emergency Medicine, 14, 96-102. http://dx.doi.org/10.5811/westjem.2012.7.6678

[12] Lovesky, D., Bird, S. and Restoccia, M. (2003) Effect of a Paramedic Pain Management Training Program on Prehospital Analgesic Use. Academic Emergency Medicine, 16, 450.

[13] Von Roenn, J.H., Cleeland, C.S., Gonin, R., Hatfield, A.K. and Pandya, K.J. (1991) Physician Attitudes and Practice in Cancer Pain Management. A Survey from the Eastern Cooperative Oncology Group. Annals of Internal Medicine, 119, 121-126. http://dx.doi.org/10.7326/0003-4819-119-2-199307150-00005

[14] French, S., Salama, N., Baqai, S., Raslavicus, S., Ramaker, J. and Chan, S. (2006) Effects of an Educational Intervention on Prehospital Pain Management. Prehospital Emergency Care, 10, 71-76. http://dx.doi.org/10.1080/10903120500366086

[15] Pollock, M.J., Brown, L.H. and Dunn, K.A. (1997) The Perceived Importance of Paramedic Skills and the Emphasis They Receive during EMS Education Programs. Prehospital Emergency Care, 1, 263-268. 
[16] Telion, C. and Carli, P. (2002) Prehospital and Emergency Room Pain Management for the Adult Trauma Patient. Techniques in Regional Anaesthesia and Pain Management, 6, 2-9. http://dx.doi.org/10.1053/trap.2002.29358

[17] Paramedics Australasia (2013) Paramedic Role Descriptions.

[18] Galinski, M., Ruscev, M., Pommerie, F., Hubert, G., Srij, M., Lapostolle, F. and Adnet, F. (2004) National Survey of Emergency Management of Acute Pain in Prehospital Setting. Annales Françaises d Anesthésie et de Réanimation, 23, 1149-1154.

[19] Fry, M., Bennetts, S. and Huckson, S. (2001) An Australian Audit of ED Pain Management Patterns. Journal of Emergency Nursing, 37, 269-274. http://dx.doi.org/10.1016/j.jen.2010.03.007

[20] Jones, G.E. and Machen, I. (2003) Pre-Hospital Pain Management: The Paramedics Perspective. Accident and Emergency Nursing, 11, 166-172. http://dx.doi.org/10.1016/S0965-2302(02)00219-9

[21] Rickard, C., O’Meara, P., McGrail, M., Garner, D., McLean, A. and Le Lievre, P. (2007) A Randomised Controlled Trial of Intranasal Fentanyl vs. Intravenous Morphine for Analgesia in the Prehospital Setting. American Journal of Emergency Medicine, 25, 911-917. http://dx.doi.org/10.1016/j.ajem.2007.02.027

[22] Lord, B. (2004) Pain Control: The Paramedic's Role in Pain Management. American Journal of Nursing, 104, 50-53. http://dx.doi.org/10.1097/00000446-200411000-00026

[23] Benedetti, C., Dickersen, E.D. and Nichols, L.L. (2001) Medical Education: A Barrier to Pain Therapy and Palliative Care. Journal of Pain and Symptom Management, 21, 360-362. http://dx.doi.org/10.1016/S0885-3924(01)00274-3

[24] Squires, J.P.M. (2004) Developing Alternative Ambulance Response Schemes: Analysis of Attitudes, Barriers, and Change. Emergency Medicine Journal, 21, 724-727. http://dx.doi.org/10.1136/emj.2004.016923

[25] Snider, J.M., Fuller, D. and Schmidt, T. (2004) The Effect of Simple Interventions on Paramedic Aspirin Administration Rates. Prehospital Emergency Care, 8, 41-45. http://dx.doi.org/10.1080/312703002211

[26] Ricard-Hibon, A., Chollet, C. and Saada, S. (1999) A Quality Control Program for Acute Pain Management in Out-ofHospital Critical Care Medicine. Annals of Emergency Medicine, 34, 738-744. http://dx.doi.org/10.1016/S0196-0644(99)70099-5

[27] Birnbaum, A.E., Bijur, P., Holden, L. and Gallagher, E.J. (2007) Randomozed Double-Blind Placebo-Controlled Trial of Two Intravenous Morphine Dosages $(0.10 \mathrm{mg} / \mathrm{kg}$ and $0.15 \mathrm{mg} / \mathrm{kg})$ in Emergency Department Patients with Moderate to Severe Acute Pain. Annals of Emergency Medicine, 49, 445-453. http://dx.doi.org/10.1016/j.annemergmed.2006.06.030

[28] Price, L.K., Brown, G., Hughes, D. and Barton, A. (2005) A Qualitative Study of Paramedic’s Attitudes to Providing Prehospital Thrombolysis. Emergency Medicine Journal, 22, 738-741. http://dx.doi.org/10.1136/emj.2005.025536 